\title{
Análise de notícias publicadas na mídia sobre a garoupa-verdadeira (Epinephelus
}

\section{marginatus)}

Analysis of news published in the media about the grouper (Epinephelus marginatus)

Análisis de las noticias publicadas en los médios de comunicación sobre la garoupa (Epinephelus marginatus)

\section{Resumo}

A finalidade desta pesquisa foi realizar uma análise de conteúdo de notícias publicadas na mídia relacionadas a garoupa-verdadeira (Epinephelus marginatus), uma espécie de peixe marinho de ocorrência natural na costa brasileira, que apresenta alto valor comercial, boa aceitação de mercado e possui pacote tecnológico parcialmente desenvolvido. Os resultados indicam que iniciativas vêm sendo realizadas para promover a sua reprodução em cativeiro e também garantir o repovoamento de algumas regiões afetadas pela pesca artesanal e industrial. Essas atividades são realizadas com o intuito de preservar e recuperar os ecossistemas marinhos e impulsionar a piscicultura marinha nacional, como alternativa ao extrativismo e a conservação da espécie que se encontra na lista de espécies ameaçadas de extinção devido a ação humana.

Palavras-chave: Conservação da espécie; Gastronomia; Turismo; Pesca extrativista; Prospecção tecnológica; Comunicação.

\begin{abstract}
The objective of this research was to carry out a news content analysis published in the media related to Epinephelus marginatus, a marine fish specie present on the Brazilian coast, which has high commercial value, good market acceptance and has a partially developed technological package. The results indicate that initiatives are being carried out to promote their reproduction in captivity and also to guarantee the repopulation of some regions affected by artisanal and industrial fishing. These activities are carried out with the aim of preserving and recovering marine ecosystems and boosting national marine fish farming, as an alternative to extractivism and the conservation of the species that is on the list of species threatened with extinction due to human action.
\end{abstract}

Keywords: Species conservation; Gastronomy; Tourism; Extractive fishing; Technological prospecting; Communication.

\section{Resumen}

Incluir o resumo em espanhol. El objetivo de esta investigación fue realizar un análisis de contenido de las noticias publicadas en los medios de comunicación relacionadas con la garoupa (Epinephelus marginatus), una especie de pez marino que ocurre naturalmente en la costa brasileña, que tiene un alto valor comercial, buena aceptación en el mercado y tiene un paquete de tecnología parcialmente desarrollada. Los resultados indican que se han llevado a cabo iniciativas para promover su reproducción en cautiverio y también asegurar el repoblamiento de algunas regiones afectadas por la pesca artesanal e industrial. Estas actividades se realizan con el objetivo de preservar y recuperar los ecosistemas marinos e impulsar la piscicultura marina nacional, como alternativa al extractivismo y conservación de la especie que se encuentra en la lista de especies amenazadas por la acción del hombre.

Palabras clave: Conservación de especies; Gastronomía; Turismo; Pesca extractiva; Prospección tecnológica; Comunicación. 


\section{Introdução}

Em 2018, a produção mundial de pescado foi de 178,5 milhões de toneladas, no qual 82,1 milhões foram resultantes da aquicultura, representada principalmente pela criação de peixes $(66,13 \%)$, sendo 47 milhões de toneladas provenientes da piscicultura continental e 7,3 milhões da piscicultura marinha (Fao, 2020). No Brasil, mesmo com uma região costeira de aproximadamente 8,5 mil quilômetros de extensão, a aquicultura marinha resume-se à criação de moluscos bivalves e de camarões, ao passo que a piscicultura marinha ainda não tem uma produção comercial representativa para a economia de pescados (Ibge, 2019; Fao, 2020).

Na última década houve significativo esforço do governo, instituições públicas de pesquisa e empresas privadas para promover a cadeia da piscicultura marinha no Brasil, contudo, a demora e as dificuldades estabelecidas para o licenciamento ambiental foram minando os ânimos e os investimentos, dificultando o desenvolvimento da atividade no país (Aquaculture Brasil, 2020). Por esse motivo, a piscicultura marinha nacional restringe-se apenas ao desenvolvimento de pesquisas em algumas instituições públicas e a pequenas produções realizadas pela iniciativa privada. Até o momento, a produção por empresas privadas é pouco significativa, mas, apesar de todo o esforço, os dados de produção ainda não aparecem nas estatísticas oficiais.

Para impulsionar a piscicultura marinha nacional, destaca-se a espécie de peixe garoupa-verdadeira (Epinephelus marginatus), pertencente à família Serranidae. Com ampla distribuição geográfica, tem ocorrência do Sul da Bahia até a Patagônia Argentina (Figueiredo et al., 1980; Irigoyen et al., 2005; Condini et al., 2013). É uma espécie marinha apreciada para consumo, possui alto valor de mercado, importância ecológica e proporciona uma atividade geradora de renda e dinamizadora da economia local (Sanches, 2006; Condini et al., 2018).

A garoupa-verdadeira é bastante utilizada pela gastronomia brasileira, sendo considerada um peixe nobre, por ter uma carne saborosa e proporcionar benefícios a saúde humana, principalmente ao apresentar baixos níveis de gordura e alto valor nutritivo (Jasmine, 2018), além de fortalecer o turismo local, ao ser apresentada como diferencial gastronômico em muitas regiões do país. Esta espécie é altamente valorizada na Ilha de Santa Catarina, devido à excelente qualidade da carne e sua importância nacional, porém nem sempre a oferta do produto corresponde a alta demanda por garoupa nesta região (Gomes \& Ludke, 2007). Desse modo, percebe-se que o turismo gastronômico beneficia os setores ligados à alimentação, favorecendo o aumento da produção devido a maior demanda, gerando novos empregos e melhorando a qualidade de vida dos moradores de regiões turísticas (Gomes et al., 2007).

Além disso, a garoupa-verdadeira é uma das espécies estampadas nas cédulas de cem reais, com o intuito de estimular o interesse da população aos animais da fauna nacional em extinção e, consequentemente, despertar para sua proteção. Inclusive, algumas plataformas de petróleo são batizadas com o nome desta espécie marinha, pois estão localizadas em regiões que fazem parte do habitat natural da garoupa-verdadeira. As homenagens têm o objetivo de reforçar a importância pela preservação da espécie, pois se acredita que ninguém preserva o que não conhece, sendo fundamental a propagação do conhecimento sobre a relevância da espécie para o ecossistema marinho.

Ainda assim, em consequência a pesca indiscriminada, a garoupa-verdadeira foi incluída na lista de espécies ameaçadas em 2014, sendo classificada como “vulnerável” (Brasil, 2014). Devido a importância desta e de outras espécies de garoupas, diferentes países têm desenvolvido pesquisas em torno das temáticas: ecologia e gestão da pesca (Andrade et al., 2003; Condini et al., 2018), reprodução e larvicultura visando sua produção comercial (Cabrita et al., 2009; Kerber et al., 2012; Cunha et al., 2013) e repovoamento em áreas degradadas (Mesa et al., 2008; Riede et al., 2017).

No Brasil, uma ONG desenvolve um projeto de conservação e gestão através do repovoamento da garoupa-verdadeira (Riede et al., 2017). Os juvenis liberados no mar são produzidos por uma empresa privada localizada em Ilha Bela - SP, a qual 
iniciou as desovas da espécie em laboratório em 2017 (Rimmer; Glamuzina, 2017). A tecnologia para a reprodução em cativeiro e a produção de formas jovens da E. marginatus já está estabelecida pela iniciativa privada (Kerber et al., 2012). Em 2017, foram produzidos comercialmente cerca de 30.000 juvenis de garoupa-verdadeira no país. Apesar do domínio da tecnologia para a produção de formas jovens, ainda não existe engorda comercial desta espécie no Brasil (Rimmer; Glamuzina, 2017). Neste momento, uma empresa está desenvolvendo tecnologia para viabilizar a engorda da garoupa-verdadeira em diferentes sistemas de criação, com o objetivo de avaliar a viabilidade da produção comercial (Kerber, 2020).

Com o intuito de potencializar a produção nacional da garoupa-verdadeira, a Empresa Brasileira de Pesquisa Agropecuária (Embrapa) vem desenvolvendo ações dentro do Projeto BRS Aqua - Ações estruturantes e inovação para o fortalecimento das cadeias produtivas da aquicultura no Brasil, a fim de direcionar às tecnologias em desenvolvimento voltadas para a garoupa-verdadeira. Dentre as atividades desenvolvidas pode-se destacar o estudo prospectivo de viveiros escavados de camarão marinho desativados em SC, vistos como possíveis locais para a engorda da garoupa-verdadeira, a construção de um laboratório de produção de formas jovens da espécie na unidade da Embrapa Tabuleiros Costeiros e estudos econômicos e regime especial aduaneiro de drawback. Essas ações servirão de suporte aos futuros investidores interessados na criação da espécie, desenvolvimento de novas tecnologias e apoio a futuras exportações da garoupa-verdadeira, respectivamente.

Devido a importância ecológica e econômica desta espécie, o objetivo deste estudo foi analisar as notícias divulgadas na mídia nos últimos oito anos relacionados a garoupa-verdadeira, analisando o conteúdo que tem sido publicado e qual sua contribuição para o reconhecimento ambiental e desenvolvimento econômico da espécie no Brasil. Sendo assim, justifica-se a realização de estudos sobre midiatização na área da piscicultura, pois são precários ou até mesmo inexistentes.

\section{Metodologia}

Este trabalho compilou as notícias divulgadas na mídia relacionadas a espécie marinha garoupa-verdadeira publicadas no período de fevereiro de 2013 a janeiro de 2021.

Com o suporte da plataforma Cortex, definiu-se as palavras-chave: garoupa or Epinephelinae marginatus para o monitoramento realizado via web mining (Figura 1). De um total de 56 notícias encontradas, utilizaram-se 52 notícias que apresentaram fundamento ao objetivo proposto por esse estudo. Assim, as outras quatro notícias foram excluídas por não terem aderência ao contexto deste estudo, duas das notícias estando relacionadas a criação de gado e a palavra "garoupa" sendo utilizada como termo técnico específico da área. As demais notícias tratavam uma sobre economia neutra de carbono e a outra referente a eletricidade, em ambas notícias o sobrenome das entrevistadas é Garoupa, por isso foi incluído na busca realizada. 
Research, Society and Development, v. 11, n. 2, e53311226227, 2022

(CC BY 4.0) | ISSN 2525-3409 | DOI: http://dx.doi.org/10.33448/rsd-v11i2.26227

Figura 1. Monitoramento de notícias divulgadas na mídia relacionadas ao peixe marinho garoupa-verdadeira.

\section{MONITORAMENTO DE NOTÍCIAS SOBRE A GAROUPA}

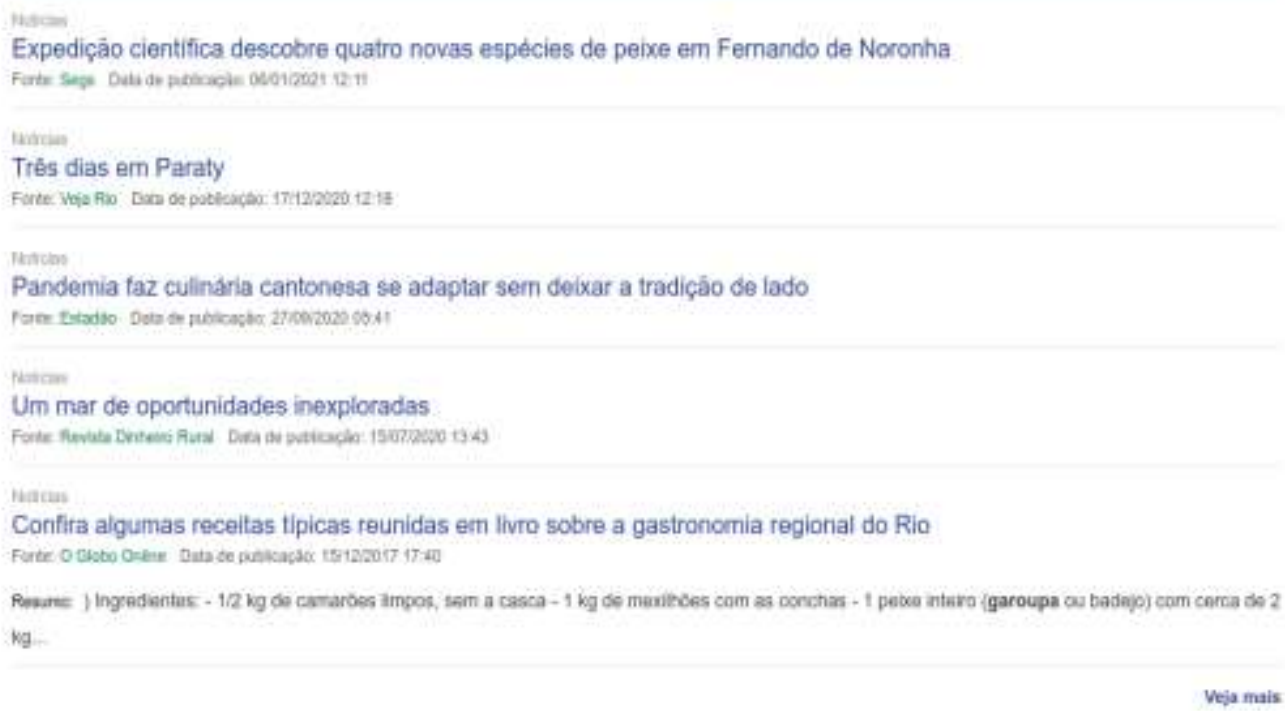

Fonte: Autores.

Para tratamento dos dados, recorreu-se a análise de conteúdo do tipo temática. Para Bardin (1977), esta é uma importante ferramenta na condução de dados qualitativos, pois se configura em um conjunto de técnicas comunicacionais que utiliza de procedimentos sistemáticos categorizados por temas e objetivos de descrição do conteúdo das mensagens.

Com isso, foram propostas sete categorias analíticas como forma do estudo ter parâmetros e identificação de variáveis sob observação, a fim de se definir com clareza pontos de comparação. Isto pode ser verificado na próxima seção.

\section{Resultados e Discussão}

Com o monitoramento das notícias divulgadas na mídia foi possível constatar que os principais temas abordados sobre a garoupa-verdadeira estão relacionados a preservação da espécie, pesca artesanal e industrial, gastronomia, turismo, consumo de peixes, homenagem à espécie como forma de conscientização de sua preservação e o desenvolvimento de tecnologias para a criação em cativeiro (Tabela 1). O perigo de extinção da espécie foi um tópico recorrente em quase todos os temas abordados, sendo essa uma questão preocupante em outras regiões do mundo onde a garoupa-verdadeira também é alvo da pesca indiscriminada, sendo mundialmente classificada como "em perigo" de extinção (Portal Fator Brasil, 2017; Segs, 2019). 
Tabela 1. Notícias relacionadas a garoupa-verdadeira encontradas na mídia entre os anos de 2013 até 2021.

\begin{tabular}{cc}
\hline Categoria das notícias & Número \\
\hline Preservação da espécie & 16 \\
Pesca Artesanal e Industrial & 9 \\
Gastronomia & 9 \\
Turismo & 7 \\
Consumo de peixes & 4 \\
Homenagem à espécie como forma de & \\
conscientização & 4 \\
Desenvolvimento de tecnologias para a criação em & 3 \\
\hline cativeiro & 52 \\
\hline
\end{tabular}

Fonte: Autores.

A espécie por fazer parte do topo da cadeia alimentar, torna-se importante para a manutenção do equilíbrio ecológico em ecossistemas recifais, regulando a abundância de suas presas, como moluscos, crustáceos e demais espécies de peixes marinhos. Isso sugere influentes modificações do ecossistema caso ocorra alteração na densidade populacional da garoupaverdadeira, sendo importante manter um equilíbrio para o seu adequado funcionamento. Este equilíbrio é diretamente interferido pelos efeitos provocados pela pesca, por isso ações de preservação da espécie são tão importantes e merecem sua devida atenção (AMDA, 2016; A Notícia - SC, 2016; Portal Fator Brasil, 2017).

A garoupa-verdadeira é um peixe muito apreciado pela gastronomia brasileira e mundial, isso se deve a qualidade da sua carne e elevado valor nutricional, que contribui para a saúde humana com o aumento do consumo de peixes na dieta alimentar, o qual vem aumentado modestamente pelos brasileiros (Exame, 2017). Apesar de ser uma espécie bem procurada em restaurantes, campanhas para incentivar o consumo de peixes alternativos aos que estão em risco de extinção são também reforçadas no país, com o intuito de diversificar o consumo de peixes e também servir como uma medida de sustentabilidade para a pesca artesanal e industrial (Akatu, 2016; Exame, 2017).

Ainda assim, a garoupa-verdadeira tornou-se o carro chefe da culinária de algumas regiões brasileiras, sendo caracterizada como prato típico e agregando valor ao turismo local. Cada vez mais restaurantes e renomados chefes de cozinha apostam na utilização da carne da garoupa para criação das mais variadas receitas, pois mesmo sendo um peixe de valor mais elevado, muitos clientes e turistas tem preferência por essa espécie. Desse modo, nota-se que a gastronomia está associada ao turismo, promovendo a divulgação de algumas regiões turísticas e preservando a cultura gastronômica local (Gomes et al., 2007; Gazeta Online, 2019; Estadão, 2020). Afinal, o turismo gastronômico é parte integrante do turismo cultural, possui grande importância para a economia local, promovendo o desenvolvimento de empresas ligadas à alimentação, gerando novas oportunidades de trabalho e melhorando a qualidade de vida dos cidadãos (Gomes et al., 2007; Veja Rio, 2020).

Contudo, a demanda para consumo da espécie é atendida exclusivamente pela pesca, a qual não consegue atender essa demanda em todas as estações do ano. Há dificuldade no fornecimento constante de matéria prima pelos pescadores e a aquisição da garoupa ainda é deficiente quando a demanda aumenta (Gomes et al., 2007). Ainda assim, a grande procura pela espécie potencializa a pesca indiscriminada, provocando alterações do ecossistema marinho e prejudicando também outras espécies existentes no mesmo habitat. A pesca da garoupa-verdadeira em algumas regiões é considerada ilegal e tem sido alvo da autuação do Instituto Brasileiro do Meio Ambiente e dos Recursos Naturais Renováveis (Ibama) por crime ambiental, sendo que este peixe marinho faz parte da lista de espécies em extinção (Famato, 2016; Diário Catarinense, 2017; Globo.com, 2018; G1, 2020). 
Com a intenção de minimizar os impactos provocados pela pesca predatória e regularizar a atividade, técnicos do Ministério do Meio Ambiente (MMA), Instituto Chico Mendes da Biodiversidade (ICMBio) e demais instituições de conservação da biodiversidade marinha vem propondo estratégias de manejo para algumas espécies que estão na lista das ameaçadas de extinção, como a garoupa-verdadeira (AMDA, 2016; A Notícia - SC, 2016; Portal Fator Brasil, 2017; Segs, 2019). Entre essas estratégias, a primeira e talvez mais importante seria:

[...] a conscientização - convencer o pescador a devolver ao mar o animal em risco o mais rápido possível, para reduzir o número de mortes. $\mathrm{O}$ desafio está em aliar medidas de proteção que também permitam uma fiscalização efetiva, considerando-se os recursos escassos de órgãos fiscalizadores como o Ibama (A Notícia - SC, 2016).

Existe uma forte preocupação por áreas com desequilíbrio ambiental, em que espécies passam a ser ameaçadas de extinção devido a ação humana, pois a recuperação das mesmas pode levar décadas ou ser irreversível. Pensando na preservação de algumas espécies, o MMA e demais instituições públicas ligadas à conservação da biodiversidade marinha vem há alguns anos discutindo propostas de manejo para espécies consideradas em situação "vulnerável". Ainda, o governo editou seis portarias para tentar preservar espécies marinhas que já estão na Lista Nacional das Espécies da Fauna Brasileira ameaçadas de extinção. Esta lista é um instrumento relevante usado pelo Ministério do Meio Ambiente para a conservação da biodiversidade (JBlogs - Jornal do Brasil, 2018; O Globo Online, 2018; Globo Rural 2019).

O maior desafio é a ausência de uma fiscalização efetiva que garanta a proteção das espécies. Atualmente os órgãos fiscalizadores como o Ibama dispõem de recursos escassos, sendo necessário solicitar equipamentos a outros instituições para executarem o trabalho de monitoramento e fiscalização da pesca (Diário Catarinense, 2017).

Com o intuito de desenvolver ações a serem realizadas em ambientes costeiros e marinhos brasileiros, projetos de proteção ao meio ambiente tem recebido apoio financeiro da Fundação Grupo Boticário de Proteção à Natureza. Estão em andamento programas de monitoramento ambiental para orientar medidas mitigadoras dos impactos identificados nas regiões afetadas. No âmbito social, algumas das ações visam conscientizar estudantes e formadores de opinião sobre a importância da conservação da garoupa-verdadeira para a manutenção dos ecossistemas marinhos (Portal Fator Brasil, 2017; Gazeta do Povo PR, 2018; O Globo Online, 2019).

Ainda, com o intuito de garantir o equilíbrio ecológico marinho e impedir que as garoupas entrem em extinção, a Associação Ambientalista Terra Viva (Atevi) com o apoio da Fundação Grupo Boticário de Proteção à Natureza, promoveu o repovoamento com 2 mil alevinos criados em cativeiro, como forma de garantir a sobrevivência da espécie na praia da Ilha das Cabras, na região sul de Ilhabela (SP), um santuário municipal onde a pesca não é permitida (Globo Rural, 2019). Segundo o coordenador de Ciência e Conservação da Fundação Grupo Boticário, Robson Capretz:

[...] a ausência das garoupas gera consequências em todo o ecossistema marinho. Assim como os tubarões e os meros, as garoupas são topo de cadeia na região que habitam e são muito importantes para manter o resto da cadeia equilibrada. Sem a sua presença, outros animais marinhos podem se reproduzir em larga escala, desequilibrando o ecossistema (Segs, 2019).

Em 2017, projetos de proteção ao meio ambiente tanto no estado de São Paulo como no Rio de Janeiro receberam apoio financeiro de mais de $\mathrm{R} \$ 640$ mil reais cada visando avaliar o padrão de conectividade da garoupa-verdadeira ao longo de sua distribuição na costa brasileira, por meio de marcadores genéticos. Ainda, o projeto prevê ações de educomunicação visando conscientizar estudantes e formadores de opinião sobre a importância da conservação da garoupa-verdadeira e dos ecossistemas marinhos (Portal Fator Brasil, 2017; Segs, 2017). 
Mesmo com investimentos em projetos para preservação de espécies em extinção em distintas regiões brasileiras e com o título de Patrimônio Mundial da Unesco dado a Paraty e Ilha Grande, animando ambientalistas sobre o reconhecimento internacional da relevância da biodiversidade e a importância no fortalecimento por melhorias na conservação do ecossistema local, o governo federal vem incentivando fortemente o turismo em regiões que são consideradas importantes devido a biodiversidade natural e que devem ser preservadas para sua manutenção. As atividades humanas podem descaracterizar o ecossistema marinho, gerando prejuízos ambientais, culturais e sociais futuramente (O Globo Online, 2019).

Dessa forma, é importante ressaltar que o turismo para ser duradouro deve estar associado ao planejamento estratégico para a conservação dos ambientes naturais, pois sem a preservação da biodiversidade não há interesse turístico local. Por isso é de suma importância o trabalho de conscientização dos pescadores, comunidade local e dos turistas, pois ninguém conserva o que não conhece e a informação sobre a importância da garoupa-verdadeira para o turismo e seu papel no ecossistema marinho pode mudar a forma de adquiri-la e ainda promover sua criação em cativeiro para um consumo consciente e sustentável.

Além disso, o investimento em pesquisas e desenvolvimento de tecnologia é primordial para o progresso da piscicultura marinha no Brasil. As zonas de mar aberto têm o potencial de produzir 15 bilhões de toneladas de peixe a cada ano, os oceanos representam uma imensa oportunidade para a produção de alimentos, mas é amplamente inexplorado como um recurso aquícola, apenas cerca de 40 espécies representam $90 \%$ da produção aquícola mundial, sendo somente $4 \%$ de peixes como salmão, perca-gigante, garoupa e robalo (Jornal de Santa Catarina, 2017). Pensando nisso, em 2018, o Instituto de Pesca inaugurou o Laboratório de Piscicultura Marinha do Núcleo de Pesquisa e Desenvolvimento do Litoral Norte (Npdln), em Ubatuba - SP, para ampliar as pesquisas sobre reprodução, larvicultura e engorda de espécies de peixes marinhos nativos do litoral brasileiro, realizando estudos com a garoupa-verdadeira e o mero, peixes de alto valor comercial e que estão ameaçados de extinção (Página Rural, 2018). Para mais explicações, Eric Routledge, pesquisador da Embrapa relata que:

[...] a maricultura pode ser tão relevante na balança comercial como são as indústrias de aves e suínos, mas isso só acontecerá se houver uma decisão do país em ser referência na produção e exportação de frutos do mar. O agronegócio se desenvolveu de costas para o mar. (Revista Dinheiro Rural, 2020).

A sócia proprietária da empresa Redemar Alevinos, Claudia Kerber, conhece bem as dificuldades para a produção de peixes marinhos no Brasil. Sua empresa foi uma das pioneiras na tentativa de produção do peixe Bijupirá em alto-mar, porém um dos problemas enfrentados foi na disputa da área marítima com outras atividades, além de roubo da produção. A produção da empresa voltou-se para a garoupa-verdadeira, no qual atualmente produz 30 toneladas de garoupas em tanques de recirculação e em viveiros de camarão, tendo como plano chegar a 100 toneladas em 2022 (Revista Dinheiro Rural, 2020). Segundo relatos de Rhyder Ramos, sócio na empresa Redemar Alevinos:

[...] a meta é ousada, mas com a produção no continente tivemos melhores resultados operacionais e também na qualidade do produto. Além disso, a garoupa verdadeira está muito valorizada na culinária brasileira e em mercados internacionais como os Estados Unidos (Revista Dinheiro Rural, 2020).

Mesmo que a demanda nacional seja conhecida, o país não supri o mercado interno regularmente e não é um exportador de garoupa e de qualquer outro peixe marinho, pois a produção em cativeiro é irrelevante (Gomes et al.,2007; Revista Dinheiro Rural, 2020). Contudo, estamos diante de uma grande oportunidade de negócios, que pode tornar o Brasil uma potência exportadora de proteína animal vindas do mar. Isso apenas será possível com um planejamento estratégico e a união das instituições públicos e privados, em busca de tecnologias para a criação em cativeiro e regularização dos licenciamentos ambientais para utilização das águas marinhas abundantes em nossa região costeira. 


\section{Conclusão}

A partir dos resultados gerados com a análise de notícias divulgadas na mídia relacionadas ao peixe marinho garoupa, conclui-se que é uma espécie de grande relevância para a gastronomia brasileira, fazendo parte da gastronomia turística de muitas regiões e tendo demanda para o seu consumo. No entanto, um dos desafios é suprir a demanda, garantindo seu fornecimento ao longo do ano. Por ser uma espécie proveniente basicamente da pesca extrativista, sua oferta fica dependente das épocas propícias e da permissão para a pesca desta espécie. A necessidade de fiscalização é legítima por se tratar de uma espécie que está em perigo de extinção e os órgãos públicos precisam reforçar sua atuação sobre a pesca ilegal da garoupa, evitando prejuízos ambientais e econômicos.

Ainda mais importante do que a fiscalização efetiva pelos órgãos competentes, é a continuidade dos programas de conscientização da pesca e consumo de peixes pertencentes a lista de animais em perigo de extinção. Além disso, investimentos no desenvolvimento de tecnologias para a produção da espécie em cativeiro são extremamente importantes. As instituições públicas e privadas precisam se unir e buscar apoio governamental para alavancar a maricultura nacional, pois sabe-se que a área costeira do país é imensa, o que nos falta é o aprimoramento de tecnologias para criação em cativeiro, apoio e investimento para o crescimento do setor. Trata-se, assim, da necessidade de o agronegócio aquícola impulsionar a atividade da piscicultura marinha, garantir o fornecimento dessa matéria prima de qualidade e contribuir com a fonte de proteína animal para o comércio nacional e internacional.

\section{Agradecimentos}

Agradecemos pelo apoio financeiro do Banco Nacional de Desenvolvimento Econômico e Social (BNDES), da Secretaria de Aquicultura e Pesca do Ministério da Agricultura Pecuária e Abastecimento do Brasil (SAP-MAPA), da Empresa Brasileira de Pesquisa Agropecuária (Embrapa) e pela parceria com Conselho Nacional de Desenvolvimento Científico e Tecnológico $(\mathrm{CNPq})$ ao projeto "BRS Aqua - Ações estruturantes e inovação para fortalecimento das cadeias produtivas da Aquicultura no Brasil".

\section{Referências}

A Notícia - SC. (2016). Impactos da lista de espécies em extinção são avaliados em estudos sobre manejo. Available at: 〈https://bit.ly/38uLsCI >.

Amda. (2016). Cientistas avaliam vida marinha em Abrolhos. Available at: <https://bit.ly/3cmB7K3>.

Andrade, A. B., Machado, L. F., Hostim-Silva, M. \& Barreiros, J. P. (2003). Reproductive biology of the dusky grouper Epinephelus marginatus (Lowe, 1834). Brazilian Archives of Biology and Technology, 46(3): 373-381. DOI: https://doi.org/10.1590/S1516-89132003000300009

Bardin, L. (1970). Análise de conteúdo. Lisboa: Edições 70.

Brasil. (2014). Ministério do Meio Ambiente. Portaria $n^{\circ} 445$, de 17 de dezembro de 2014. Reconhecer como espécies de peixes e invertebrados aquáticos da fauna brasileira ameaçadas de extinção aquelas constantes da "Lista Nacional Oficial de Espécies da Fauna Ameaçadas de Extinção - Peixes e Invertebrados Aquáticos".

Cabrita, E., Engrola, S., Conceição, L. E. C., Pousão-Ferreira, P. \& Dinis, M.T. (2009). Successful cryopreservation of sperm from sex-reversed dusky grouper, Epinephelus marginatus. Aquaculture, 287(1-2):152-157. DOI: https://doi.org/10.1016/j.aquaculture.2008.10.019

Condini, M. V., Fávaro, L. F., Varela Jr., A. S. \& Garcia, A. M. (2013). Reproductive biology of the dusky grouper (Epinephelus marginatus) at the southern limit of its distribution in the south-western Atlantic. Marine and Freshwater Research, 65(2):142-152. DOI: https://doi.org/10.1071/MF13120

Condini, M.V., García-Charton, J. A. \& Garcia, A. M. (2018). A review of the biology, ecology, behavior and conservation status of the dusky grouper, Epinephelus marginatus (Lowe 1834). Reviews in Fish Biology and Fisheries, 28(301):301-330. DOI: https://doi.org/10.1007/s11160-017-9502-1

Cunha, M. E., Ré, P., Quental-Ferreira, H., Gavaia, P. J. \& Pousão-Ferreira, P. (2013). Larval and juvenile development of dusky grouper Epinephelus marginatus reared in mesocosms. Journal of Fish Biology, 83(3):448-465. DOI: https://doi.org/10.1111/jfb.12180

Diário Catarinense. (2017). Multas aplicadas pelo Ibama em SC aumentam em $400 \%$ no início do ano. 〈http://bit.ly/2N3ZmEe >.

Estadão. (2020). Pandemia faz culinária cantonesa se adaptar sem deixar a tradição de lado. <https://bit.ly/3byZsxh > . 
Research, Society and Development, v. 11, n. 2, e53311226227, 2022

Exame. (2017). Conheça os benefícios de 40 tipos de peixe. 〈http://bit.ly/3bysDjS〉. 2021.

Famato. (2016). Pesca industrial ganha força no Brasil. < https://bit.ly/3cc6Q0k>.

Fao - Food and Agriculture Organization of the United Nations. (2020). The State of World Fisheries and Aquaculture 2020. Sustainability in action. Rome: FAO. <https://doi.org/10.4060/ca9229en>.

Figueiredo, J. L. \& Menezes, N. A. (1980). Manual de peixes marinhos do sudeste do Brasil. São Paulo: Editora Museu de Zoologia da Universidade de São Paulo. 90 p.

G1. (2020). Operação contra pesca ilegal em Laguna resulta em multa milionária. < http://bit.ly/2OJKcEs>.

Gazeta do Povo - PR. (2018). Pode ser 'pecado' comer alguns peixes na Sexta-Feira Santa - inclusive o bacalhau. < https://bit.ly/3qB8Nsv>.

Gazeta Online. (2019). Prato tipicamente capixaba, aprenda a fazer arroz de garoupa salgada. 〈http://bit.ly/3120CV3>.

Globo Rural. (2019). Operação contra extinção da garoupa introduz 2 mil alevinos em mar de SP. < http://glo.bo/3cnAZde>.

Globo.com. (2018). Governo excluiu aos 45 do $2^{\circ}$ tempo' áreas mais vulneráveis de reservas marinhas, dizem pesquisadores. Available at: < http://glo.bo/3ciNOR9>.

Gomes, A. A. \& Ludke, E. N. (2007). Estudo de viabilidade da utilização da garoupa como diferencial na gastronomia da ilha de Santa Catarina. Trabalho de Conclusão de Curso (Turismo) - Universidade do Sul de Santa Catarina, Florianópolis, SC.

Jasmine. (2018). Os melhores peixes para a manutenção da saúde. < http://bit.ly/30wMbyQ.>.

Ibge - Instituto Brasileiro de Geografia e Estatística. (2019). Pesquisa da Pecuária Municipal: Produção da aquicultura, por tipo de produto. <https://sidra.ibge.gov.br/tabela/3940>.

Irigoyen, A. J., Galván, D. E. \& Venerus, L.A. (2005). Occurrence of dusky grouper Epinephelus marginatus (Lowe, 1834) in gulfs of northern Patagonia, Argentina. Journal of Fish Biology, 67(6):1741-1745. DOI: https://doi.org/10.1111/j.1095-8649.2005.00866.x

Jblogs - Jornal do Brasil (2018). Portarias para espécies marítimas. Available at: < https://bit.ly/3qE8VaP>.

Jornal de Santa Catarina. (2017). Fazendas em alto mar podem ajudar a alimentar população em expansão. < http://bit.ly/2OJYVze>.

Kerber, C. E. (2020). Avanços recentes no cultivo de garoupas no Brasil. In: BEGOSSI, A. \& LOPES, P. F. M. (Orgs.). Garoupa e pescadores (Epinephelus marginatus). (pp. 89-97). São Carlos: Editora RiMa,.

Kerber, C. E., Silva, H. K. A., Santos, P. A. \& Sanches, E. G. (2012). Reproduction and larviculture of dusky grouper Epinephelus marginatus (Lowe 1834) in Brazil. Journal of Agricultural Science and Technology, 1:229- 234.

Mesa, G. L., Longobardi, A.,Sacco, F. \& Marino, G. (2008). First release of hatchery juveniles of the dusky grouper Epinephelus marginatus (Lowe, 1834) (Serranidae: Teleostei) at artificial reefs in the Mediterranean: results from a pilot study. Scientia Marina, 72(4):743-756. DOI: https://doi.org/0.3989/scimar

Mpa/Mma - Ministério da Pesca e Aquicultura e Ministério do Meio Ambiente. (2012). Plano de gestão para o uso sustentável da tainha, Mugil liza Valenciennes, 1836, no Sudeste e Sul do Brasil. Proposta elaborada pelo grupo técnico de trabalho-GTT tainha, instituído pela Portaria Interministerial ${ }^{\circ} 1$, de 28 de junho de 2012 .

O Globo Online. (2018). Projeto aposta na captação em tempo seco para melhorar qualidade das águas da Guanabara. 〈http://glo.bo/3t5XutT〉.

O Globo Online. (2019). Unidade ambiental que Bolsonaro quer transformar em "Cancún" é obrigatória para funcionamento de usina nuclear. < https://glo.bo/3veJtvS>.

Akatu. (2016). Como fisgar o consumidor para salvar espécies marinhas da sobrepesca. 〈https://bit.ly/3cffsU6>.

Página Rural. (2018). São Paulo: piscicultura ganha força e competitividade com incentivo a pesquisa e desburocratização das atividades. < https://bit.ly/3qANCab>.

Portal Fator Brasil. (2017). Projetos de proteção do meio ambiente no Rio de Janeiro receberão apoio de $R \$ 647$ mil. $<\mathrm{https} / / / \mathrm{bit} .1 \mathrm{y} / 3 \mathrm{qE} 9 \mathrm{bXl}\rangle$.

Revista Dinheiro Rural. (2020). Um mar de oportunidades inexploradas. < http://bit.ly/2N3Scji >

Riede, R., Kerber, C., Correal, G., Mata Jr., M. \& Castro-Cardoso, F. (2017). Captive-reared dusky grouper (Mycteroperca marginata) as an alternative to repopulation of degraded reef habitats. Journal of Fisheries and Aquaculture Development, 3:1-5.

Rimmer, M. A. \& Glamuzina, B. (2017). A review of grouper (Family Serranidae: Subfamily Epinephelinae) aquaculture from a sustainability Science perspective. Reviews in Aquaculture, 11:58- 87. DOI: https://doi.org/10.1111/raq.12226

Sanches, E. G. (2006). Perspectivas para o cultivo de garoupas e badejos no Brasil. Pesquisador do Núcleo de Pesquisa e Desenvolvimento do Litoral Norte, Centro APTA do Pescado Marinho, Instituto de Pesca - SAA - São Paulo.

Segs. (2017). Projetos de proteção do meio ambiente em São Paulo receberão apoio de $R \$ 656$ mil. 〈http://bit.ly/314FIET〉.

Segs. (2019). Projetos terão apoio de cerca de $R \$ 650$ mil para preservar áreas e espécies litorâneas no Sudeste do Brasil. $\langle$ http://bit.ly/3vb26B1>.

Aquaculture Brasil. (2020). Terra à Vista! O Início da Piscicultura Marinha Brasileira em Ras. <https://www.aquaculturebrasil.com/coluna/118/terra-a-vista-oinicio-da-piscicultura-marinha-brasileira-em-ras>.

Veja Rio. (2020). Três dias em Paraty. < http://bit.ly/313ve8A>. 